\title{
Properties of nematic LC planar and smoothly-irregular waveguide structures: research in the experiment and using computer modeling
}

\author{
A.A. Egorov ${ }^{1}$, L.A. Sevastyanov ${ }^{2,3}$, V.D. Shigorin ${ }^{1}$, A.S. Ayriyan ${ }^{3,4}$, E.A. Ayriyan ${ }^{3}$ \\ ${ }^{I}$ A.M. Prokhorov General Physics Institute, Russian Academy of Sciences, Moscow, Russia; \\ ${ }^{2}$ Peoples' Friendship University of Russia (RUDN University), Moscow, Russia; \\ ${ }^{3}$ Joint Institute for Nuclear Research, Dubna, Moscow region, Russia; \\ ${ }^{4}$ A.I. Alikhanyan National Science Laboratory, Yerevan, Armenia
}

\begin{abstract}
Nematic liquid crystal planar and smoothly-irregular waveguide structures were studied experimentally and by the computer modeling. Two types of optical smoothly-irregular waveguide structures promising for application in telecommunications and control systems are studied by numerical simulation: liquid crystal waveguides and thin film solid generalized waveguide Luneburg lens. Study of the behavior of these waveguide structures where liquid crystal layer can be used to control the properties of the entire device, of course, promising, especially since such devices are also able to perform various sensory functions when changing some external parameters, accompanied by a change in a number of their properties. It can be of interest to researchers not only in the field of the integrated optics but also in some others areas: nano-photonics, optofluidics, telecommunications, and control systems. The dependences of the attenuation coefficient (optical losses) of waveguide modes and the effective sizes (correlation radii) of quasi-stationary irregularities of the liquid-crystal layers on the linear laser radiation polarization and on the presence of pulse-periodic electric field were experimentally observed. An estimate was made of the correlation radii of liquid-crystal waveguide quasi-stationary irregularities. The obtained results are undoubtedly important for further research of waveguide liquid crystal layers, both from the theoretical point of view, and practical - in the organization and carrying out new experimental researches, for example, when developing promising integrated-optical LC sensors.

Keywords: waveguide, planar lens, smoothly-irregular, liquid crystal, laser, director, irregularities, optofluidics, sensor, numerical simulation.

Citation: Egorov AA, Sevastyanov LA, Shigorin VD, Ayriyan AA, Ayriyan EA. Properties of nematic LC planar and smoothly-irregular waveguide structures: research in the experiment and using computer modeling. Computer Optics 2019; 43(6): 976-982. DOI: 10.18287/2412-61792019-43-6-976-982.
\end{abstract}

\section{Introduction}

In this paper we presented the results of studying the integrated optical waveguides and thin-layer waveguide lenses based on the nematic liquid crystal (NLC) [1, 2], in particular, their behavior under the high-power pulsedperiodic electric field [3-8]. The study of the behavior of such combined structures when changing some external parameters, accompanied by a change in a number of their properties, is undoubtedly of interest to researchers not only from this subject area, since the appearance of new properties and characteristics may be of practical interest as in the integrated optics and also in other areas, e.g. in optofluidics, nano-photonics, telecommunications, monitoring systems of environmental state parameters, etc (see e.g. $[1-13])$.

Liquid crystals (LC) structure is a viscous fluid consisting of molecules of elongated or disk-like shape, definitely arranged in the entire volume of this liquid $[1,2]$. The most characteristic property of liquid crystals is their ability to change the orientation of molecules under the influence of electric or magnetic fields, which opens up wide opportunities for their use in various fields of science, technology and industry, including integrated optics and integrated optoelectronics (see, e.g. [1-13]).

The progress of technology stimulates further interest to the development and improvement of integrated optical and fiber optical sensors and integrated processors, intended for the use in different fields of science, engineering, and industry, particularly, in promising telecommunication technologies and in different control systems (see, e.g. [5, 12-14]).

The basis of most of the electro- and magneto-optical effects specific for LC is the director's (the axis of the predominant direction of molecules of a macroscopic volume of matter) reorientation under the action of a field or a fluid flow. The result of reorientation is the change in the optical properties of the medium. This process of reorienting the director (local or taking place throughout the sample as a whole) can be traced in all electro- and magneto-optical effects. The NLC does not have a layered structure in the initial state; their molecules glide continuously in the direction of their long axes, rotating around them, but retain a long-range orientation order: the long axes are directed along one preferred direction.

One of the major stages of developing an optical integrated system is the analysis and synthesis of the optical components, necessary for its normal functioning, by means of computer modeling and computer-aided design with the usage of modern numerical methods (see, e.g. [15-27]).

\section{Experimental setup. Waveguiding NLC structures}

The objects of the study are the integrated optical waveguide (Fig. 1, 2) formed from NLC 4-Cyano-4'- 
pentylbiphenyl (4-Cyano-4'-pentylbiphenyl or 5CB), well known from publications in the scientific and technical literature, as well as planar waveguide NLC lenses (Fig. 2).

In Fig. 1 is depicted: 1 is the laser polarized focused beam, 2 is LC layer, 3 is the LC cell, 4 is the metal (copper) electrodes, 5 is the LC cell retainer, 6 is the glass plate on which LC cell is fixed. Scattering in NLC waveguide radiation is registered in the camera situated above LC cell, and then the signal enters in the computer.

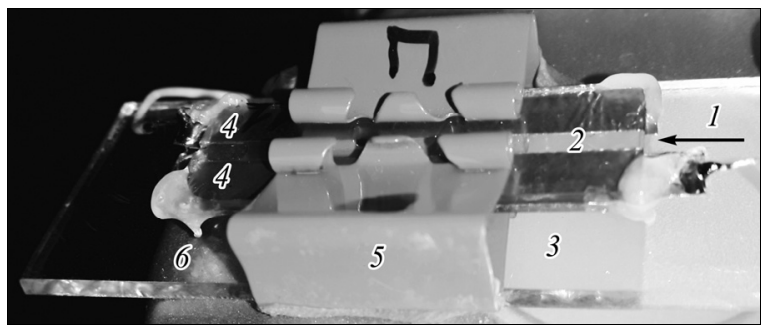

Fig. 1. Schematic representation of the experimental cell

(a)

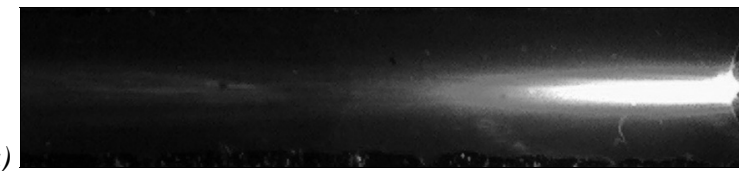

(b)

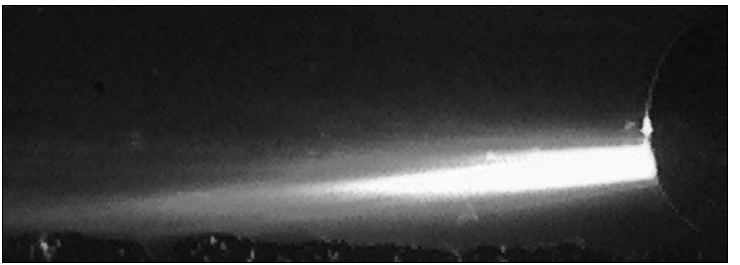

Fig. 2. Photograph of tracks of waveguide modes: (a) left and center of the photo - in the integrated optical NLC waveguide; at the right-planar NLC lens; (b) the focusing effect of a planar NLC lens when the incident beam is shifted to the left of the center. Dimensions on the areas shown in the photo in width are about $3 \mathrm{~mm}$, and in length do not exceed 9-15 $\mathrm{mm}$

In Fig. 2 on the right at the edge of the photograph is the planar NLC lens formed at the end of the waveguide. This lens performs the role both a matching and a focusing element. Immediately behind the NLC lens, one can clearly see the characteristic beam of radiation focused on it and the tracks of the modes. Lens dimensions: radius is $1.5 \mathrm{~mm}$, thickness in the center was determined by the thickness of NLC waveguides (see below). As can be seen from the photos, the wide laser beam falling on the right is focused on a diffraction limited spot in the NLC waveguide layer.

\section{Results of the study of NLC structures}

In the experiments multimode NLC waveguides, formed by two glass plates and LC layer between them (Fig. 1), were studied. NLC had a homogeneous planar orientation with an optical axis along the direction of the director (coincides with the axis $z$ ).

Waveguides with different thicknesses $(25,75$, and $125 \mu \mathrm{m})$ were studied in experiments, but the main part of the results is given only for NLC waveguides with $h=25$ and $75 \mu \mathrm{m}$. Distance between copper electrodes was $0.2 \mathrm{~cm}$. A high-voltage pulse (repetition frequency of $10 \mathrm{~Hz}$ ) was applied to them. The NLC layer had refractive indices: ordinary $\approx 1.53$ and extraordinary $\approx 1.70$ (for the wavelength of laser radiation $\lambda \approx 0.64 \mu \mathrm{m}$ and temperature $\approx 25^{\circ} \mathrm{C}$ ). The glass plates had refractive indices: $n_{1}=n_{3} \approx 1.52$. Thus, in experiments and numerical calculations, three-layer symmetric waveguides with the following refractive indices were studied: $n_{1}=n_{3} \approx 1.52$, $n_{2} \approx 1.53$. The input efficiency of the laser radiation into NLC waveguides did not exceed $35 \%$.

The 3D "color" (grey scale) map surface of the dependence of the scattered laser radiation $I(z, y)$ for TE- and TM-polarization right after the NLC lens were also registered. The field $E$ were switched off and then switched on. We plan to present these pictures after processing and analysis in one of our next work.

We considered the propagating and scattering of the guided modes on an extended bulk 3D NLC inhomogeneity (insert type) of the nonabsorbing or absorbing waveguide layer. The research three-layer waveguide was formed e.g. by two glass plates and the nematic liquid crystal layer between them (symmetric multimode NLC waveguide), or three-layer waveguide was formed by thin layer of the liquid crystal deposited on the glass substrate (asymmetric multimode NLC waveguide), i.e. in this case the LC waveguide lens was made before the planar waveguide face (see Fig. 1).

In order to quantify the optical losses in the waveguide, computer photometry of photographs of the corresponding mode tracks was carried out. To determine the optical losses $\alpha$, we used the well-known expression:

$$
I(z)=I_{0} \exp (-\alpha z),
$$

where $I(z)$ is the intensity of radiation (per unit area) at any point along the length of the waveguide, $I_{0}$ is the intensity at the distance $z=0$ along the modes tracks, $\alpha$ was defined in $\mathrm{cm}^{-1}$. Then optical losses were determined according to formula:

$$
\alpha=\frac{1}{z} \ln \left(\frac{I_{0}}{I(z)}\right),
$$

where $z \approx 0.3 \div 0.5 \mathrm{~cm}$.

Let's give an example of the results of computer processing of data for some of the researched multimode NLC waveguides both for TE and TM-polarization (see Fig. 3, Fig. 4, Fig. 5, and Fig. 7).

TE-polarization, $h=75 \mu \mathrm{m}$, without the external electric field $E: \bar{\alpha}_{\mathrm{TE}}=4.5 \mathrm{~cm}^{-1}, r_{\mathrm{TE}} \approx 0.15 \div 0.6 \mu \mathrm{m}$; with the field $E: \bar{\alpha}_{\mathrm{TE}}=3 \mathrm{~cm}^{-1}, r_{\mathrm{TE}} \approx 0.1 \div 0.4 \mu \mathrm{m}$.

TM-polarization (see Fig. 3), $h=75 \mu \mathrm{m}$, without the field $E: \bar{\alpha}_{\mathrm{TM}}=6 \mathrm{~cm}^{-1}, r_{\mathrm{TM}} \approx 1.0 \div 1.8 \mu \mathrm{m}$; with the field $E$ : $\bar{\alpha}_{\mathrm{TM}}=5 \mathrm{~cm}^{-1}, r_{\mathrm{TM}} \approx 0.5 \div 1.5 \mu \mathrm{m}$. Here $r_{\mathrm{TE}}$ and $r_{\mathrm{TM}}$ are $r_{e f}$ for each polarization (see formula (3)).

For NLC waveguide with $h=25 \mu \mathrm{m}$ similar results were obtained, which allowed us to assume that the multimode NLC waveguides under study are of the same type and we can consider the results mainly for some of them, for example, for NLC waveguide with $h=75 \mu \mathrm{m}$. In cases where the need arises, we will specifically note these different types of multimode NLC waveguides. 
The size of the NLC irregularities was estimated in accordance with the Rayleigh criterion. Having determined the losses $\alpha$ of the optical power in the NLC waveguide in accordance with formula (2), it is possible to estimate the effective size $r_{e f}$ of the irregularities of the LC layer in accordance with the approximate formula [4]:

$$
r_{e f} \approx \lambda\left(\alpha h_{e f}\right)^{1 / 2} C(\theta),
$$

where $C(\theta)$ is the dimensionless correction factor.

We used formula (3) to obtain estimates for a number of NLC waveguides in accordance with the Rayleigh criterion, showed that the dimensions of irregularities in the NLC layer vary in the range from about 0.08 to $2.00 \mu \mathrm{m}$, and the error in the determination does not exceed $15-20 \%$.

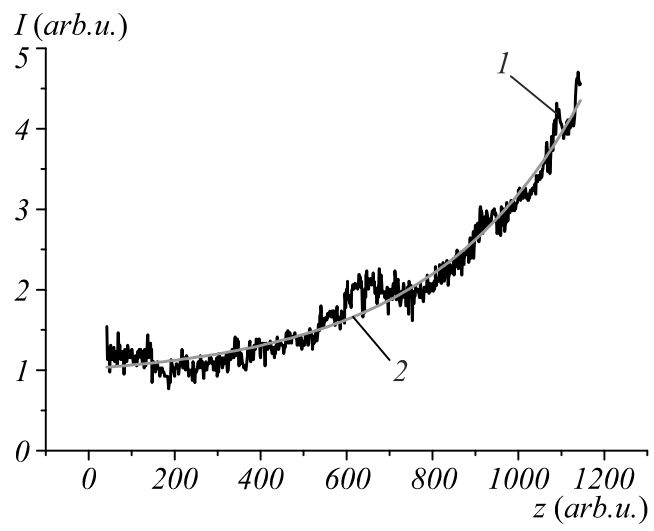

Fig. 3. Dependence of the intensity I of the scattered laser radiation on the coordinate $z(1$ arb.u. $\approx 3.5 \mu \mathrm{m})$ with the field $E$ switched on. TM-polarization. The numbers denote: 1 is the experimental curve, 2 is the fitting exponential curve

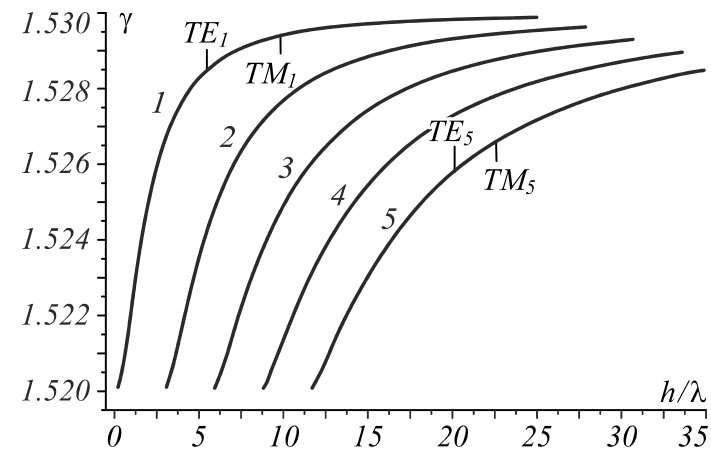

Fig. 4. Dispersion relations for the first five TE and TM modes of NLC waveguide; $\gamma$ is the effective refractive index of nematic liquid crystal waveguide

It is important to note that the maximum values of the attenuation coefficient in some liquid-crystal waveguides reached values of about $10 \mathrm{~cm}^{-1}$ and more. The waveguide sections with such large losses were not studied in detail.

To explain the established patterns of light scattering in the NLC waveguides, fluctuations in the local orientation of LC molecules were considered [1-4]. Fluctuations of the director are accompanied by elastic deformation of the medium, which leads to local random changes in the optical properties of the medium, which cause the scattering of light. In this case, the correlation radius for the director fluctuations depends inversely on the magnitude of the applied field [1].

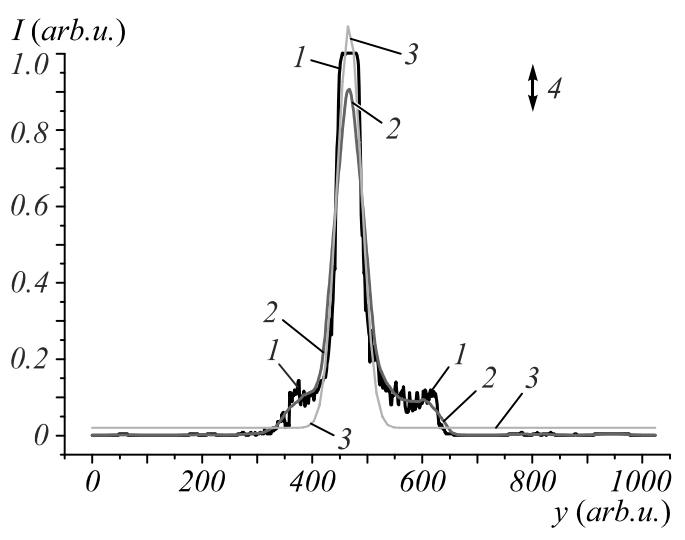

Fig. 5. The intensity profile of laser radiation behind the planar NLC lens. $\Delta \mathrm{I}_{0.7} \leq 50 \lambda$

The known laws governing the scattering of light in a non-waveguide case are explained on the basis of the consideration of fluctuations in the local orientation of the molecules, i.e. director of NLC. As a result, the radius of correlations $\xi$ of the director fluctuations can be calculated, which shows the distance at which the order given by the wall (the waveguide substrate) is stored, for example, when the field $E$ is applied (the bigger the field, the order is preserved at a smaller distance from the wall) (see e.g. $[3,4]$ ). It can be put in correspondence with the found correlation radius of the irregularities of the NLC waveguide.

Let us compare the regularity of the change in the radius of correlations of the director fluctuations $\xi \propto E^{-1}$ with the results obtained by us for changing the correlation radius of the irregularities of the NLC waveguide without the field and with the field: the correlation radius when the external field is switched on decreases for both types of modes.

This indicates a good agreement between our results and the classical theory of director fluctuations in LC [1, 3, 4]. Thus, the obtained estimate confirms the correctness of the experimental dependences found by us. Since

$$
I(z) \propto \exp (-\alpha z) \propto \exp \left(-r_{e f} z\right),
$$

then, as the correlation radius decreases, increases $I$ and the damping coefficient $\alpha$ decrease, which was discovered in the experiments.

The use of the phenomenon of waveguide scattering made it possible to substantially increase the resolution by the correlation radii in comparison with the classical methods such as the optical microscopy. In addition, the waveguide method made it possible to obtain statistical information on waveguide irregularities in one measurement with a sufficiently large volume of the waveguide layer.

The advantage of this method is also the possibility of investigating waveguide irregularities in a wide range of changes in their lateral dimensions, including the size of the order of the wavelength of the probing radiation, as in the Mie's scattering theory (see e.g. [14-23]).

In the further research we plan to use in the numeric analysis the analytic solutions of the problem of waveguide propagation, transforming and scattering of electromagnetic monochromatic radiation in an irregular 3D 
integrated-optical waveguide that we obtained previously (see e.g. [17-27]).

Fig. 5 shows one of the radiation intensity profiles measured near the back focal plane of one of the studied planar NLC lenses (curve 1). In Fig. 5 are also indicated: 2 is the curve obtained by smoothing the original distribution $1 ; 3$ is the fitting Gaussian curve; 4 is the error (less than 7\%).

The half-width of the distributions of type 1 in the vicinity of the focal plane, measured at the 0.5 level of its maximum (i.e. $\left.\Delta I_{0.5}\right)$, reached $(10-45) \lambda$, which is apparently caused by aperture restriction (small numeric aperture NA of the researched LC lenses) and also due to the fact that multimode (14 or more modes, see Fig. 4) LC waveguides were investigated [3-5].

We have compared also our measured half-width $\Delta I_{0.5}(y)$ with the half-width intensity profile at the focal distance, including the theoretical diffraction limited spot, given in the paper [9], which has a certain meaning in our case.

We can draw a conclusion, that, on the one hand, these sizes $\Delta I_{0.5}$ are close enough, and on the other hand, both of them in several times $(>3-6)$ more than the theoretical diffraction limited spot $\delta[28-30]$ :

$$
\delta=0.61 \frac{\lambda}{N A},
$$

where numeric aperture $N A<1$.

The estimation of the focal length of the studied liquid-crystal lenses has shown that it is approximately in the range from $2 R$ to $8 R$, where $R$ is the radius of the studied planar and smoothly-irregular LC lens. The results of the preliminary study did not allow making unambiguous conclusions about the effect of the linear polarization of laser radiation and the external pulseperiodic electric field on the parameters of the planar and smoothly-irregular NLC lenses under investigation. At the same time, it was unequivocally concluded that this influence is obvious and further research is needed in this direction.

In [27] one can see the plots of three-dimensional synthesized thickness profile $h(y, z)$ of the Luneburg thinlayer waveguide solid lens.

The studied Luneburg lens has the following structure: it is fabricated on a silicon substrate $\left(\mathrm{SiO}_{2}\right)$, coated with the first (regular) waveguide layer (the Corning 7059 glass), over which the second waveguide layer $\left(\mathrm{Ta}_{2} \mathrm{O}_{5}\right)$ having the variable thickness $h(y, z)$ was applied. The covering layer was air. At this stage of the research, we have not numerically studied such Luneburg NLC lens, since it is very difficult to made it experimentally. Therefore, we have mentioned this profile to show how a profile similar to it will look like in the case of the smoothly-irregular LC lenses.

We should note that this solid Luneburg lens with the profile depicted e.g. in [27] (focal length $F=7.5 \mathrm{~mm}$ and $R=5 \mathrm{~mm}$ ) executes the necessary amplitude-phase transformation with superresolution, exceeding the classical diffraction limit $\delta$ defined in the formula (4) (see e.g. [20, $26,27])$, because

$$
\Delta I_{0.5}=|\operatorname{Re}(\mathbf{E})|^{2}<0.5 \lambda .
$$

The thin-film waveguide generalized Luneberg lens is a three-layer regular waveguide on which an additional (fourth) waveguide layer (with refractive index $n_{4}$ ) of varying thickness $h(r)$ is applied, cylindrically distributed in a circle of radius $R(r \in[0, R])$. At the same time, an additional waveguide layer ensures the distribution of the effective refractive index of waveguide $\gamma(r)$, which is satisfies outside the radius $R$ the next equation [20, 26, 27]:

$$
\gamma(r) / \gamma=\exp [\omega(\rho, F)],
$$

where

$$
\rho=r \frac{\gamma(r)}{\gamma}, \omega(\rho, F)=\frac{1}{\pi} \int_{\rho}^{1} \frac{\arcsin (x / F)}{\left(x^{2}-\rho^{2}\right)^{1 / 2}} \mathrm{~d} x .
$$

Thus, the focusing effect of the generalized Luneberg lens (5) is not achieved due to the gradient of the refractive index of the lens material, as in conventional volumetric Luneberg lenses, but by changing the thickness $h(r)$, which is accompanied by a corresponding change in the effective refractive index $\gamma(r)$, while $n_{4}=$ const. That is why waveguide generalized Luneberg lens was chosen as the object of comparison with NLC lenses.

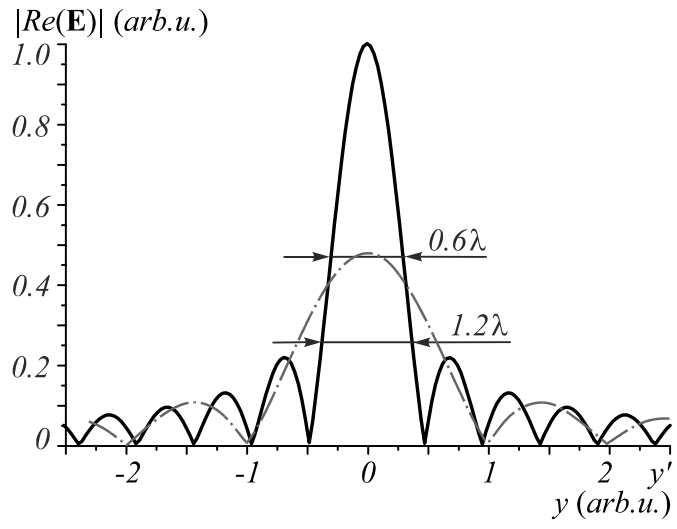

Fig. 6. Distribution of the electric field in the focal plane of the Luneburg waveguide lens presented on the Fig. 7. The solid line corresponds to accounting for $99 \%$ of the lens aperture, and the dot-dash line corresponds to $60 \%$ of the lens aperture; $y^{\prime}=y / \lambda$

We have numerically simulated propagation of eigenmodes through a Luneburg waveguide lens within the previously obtained analytical solution of the vector electrodynamic problem for a smoothly irregular fourlayer 3D integrated-optical waveguide. The programmes for numerical solution were developed in Maple and Delphi $[3-5,19,20,25-27]$. The corresponding dispersion relation has been calculated, in particular, taking into account the shift of the propagation constants of hybrid modes. The vertical distribution of the electromagnetic field of a smoothly deformed mode in a Luneburg waveguide lens has been constructed.

A full-aperture Luneburg waveguide lens has been synthesized in the zero-order vector approximation. The methods considered here make it possible to perform (in the design stage) high-precision computer analysis of all features of operation of complex multilayer smoothly ir- 
regular integrated optical 3D elements of the Luneburg waveguide lens type that are most important for experimenters. An undoubted advantage of the theoretical description, methods, and algorithms is that they can be generalized to smoothly irregular integral 3D structures composed of layers of dielectric or magnetic materials, materials with nonlinear properties, or metamaterials (see e.g. [19, 20, 25-27, 35-37].

One and two-dimensional model of Frederiks effect were used for the investigation of the electric field effect on nematic liquid crystal director orientation (see Fig. 7) in the side-electrode cell that helped us to understand the behavior of NLC (see e.g. [4, 6-8, 31]). The solutions were obtained by the standard finite-difference methods. The programmes for numerical solution of two-dimensional parabolic partial differential equation were developed both in FORTRAN, and $\mathrm{C} / \mathrm{C}++[4-8]$.

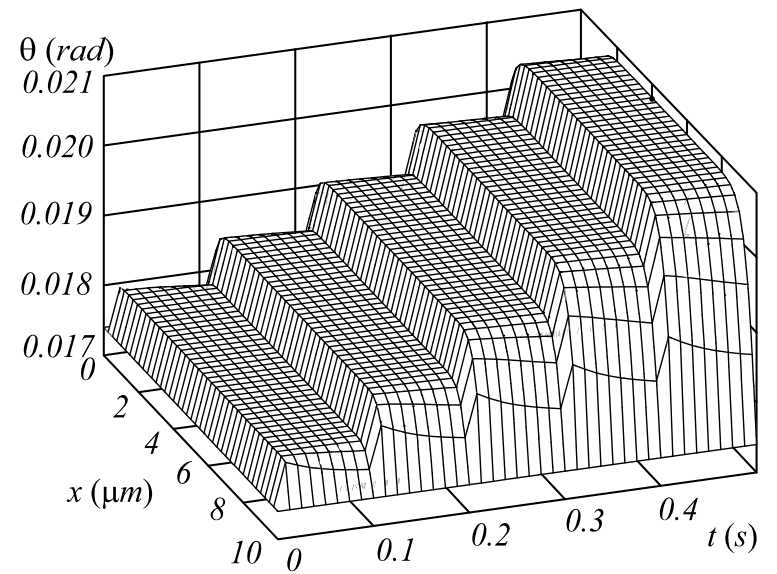

Fig. 7. 3D-dynamics of the profile $\Theta$

As one example of the computer modeling one can see on the Fig. 7 3D-dynamics of the angle $\Theta(x ; t)$ for the first 5 impulses of the external pulsed-periodic electric field $E(t)$ (pulse repetition period $T=0.1 \mathrm{~s}$ ), where $\Theta$ is the deformation angle of the director (local molecular orientation) in the NLC waveguide sell (see e.g. [3-7]). While there is a characteristic "accumulation effect":

$$
\Theta_{t \rightarrow \infty} \rightarrow \Theta_{\max }<86^{\circ} \text {. }
$$

The results of the calculations were compared to the experiments and in most cases a good match was obtained (see e.g. [3-8, 31]). Details are beyond the scope of this work. Specific details of the results of the research of Frederiks phenomenon can be found in $[1-8,31]$.

\section{Conclusion}

Nematic liquid crystal waveguiding structures were researched by the numeric simulation and experimentally. The dependence of the attenuation coefficient of the waveguide modes and the sizes of the quasi-stationary irregularities of the liquid-crystal layer on the linear polarization of the incident laser radiation and the presence of a pulseperiodic electric field is experimentally observed. An estimate is made of the correlation radii of liquid-crystal waveguide irregularities. The observed decrease in the damping coefficient of the waveguide modes and the dimensions of irregularities in the liquid-crystal layer, when the external field is switched on, explained by the effect of a decrease in the fluctuations correlation radius of the local orientation of the molecules of the liquid crystal. We have also to emphasize that the non-stationary NLC waveguide structures under study exhibited occurrence of many mode tracks whose damping coefficient (and, correspondingly, track brightness) fluctuated with time, resulting in temporal variations in their parameters which creates certain difficulties in carrying out.

The significance of these studies is provided by the practical use and prospects of using liquid crystal materials in various modern integrated-optical devices, for example, in coupling elements, modulators, distributed Bragg reflectors, sensors, processors, etc (see e.g. [3-5, 10-13, 32-38]).

The results obtained are important for further investigation of dynamic processes inside non stationary waveguide liquid crystal layers, both from the theoretical point of view for understanding kinetic processes in the liquid crystals, and practical - in the organization and carrying out appropriate experimental and theoretical researches in the field of the optofluidics and waveguiding optics.

\section{Acknowledgment}

The publication has been prepared with the support of the "RUDN University Program 5-100" (Sevastyanov L.A.) and funded by RFBR according to the research projects No. 18-07-00567, No. 18-51-18005 and No. 19-0100645.

We are grateful to: I.A. Maslyanitsyn (for his help in carrying out the experiment), I. Marinov and L. Popova (for preparing samples). We also thank G. Andler for participating in a fruitful discussion.

\section{References}

[1] Blinov LM. Electro- and magnetooptics of liquid crystals [In Russian]. Moscow: Nauka; 1978.

[2] Khoo IC. Liquid crystals. $2^{\text {nd }}$ ed. Wiley; 2007. ISBN: 9780-471-75153-3.

[3] Egorov A, Sevastianov L, Shigorin V, Andler G, Ayriyan A, Ayriyan E. Experimental and numerical study of properties of nematic liquid crystal waveguide structures. ICUMT 2018: 448-452. DOI: 10.1109/ICUMT.2018.8631282.

[4] Egorov AA, Shigorin VD, Ayriyan AS, Ayryan EA. Study of the effect of pulsed-periodic electric field and linearly polarized laser radiation on the properties of liquid-crystal waveguide. Physics of Wave Phenomena 2018; 26(2): 116123. DOI: $10.3103 / \mathrm{S} 1541308 X 18020012$.

[5] Egorov AA, Andler G, Sevastyanov AL, Sevastyanov LA. On some properties of smoothly irregular waveguide structures critical for information optical systems. Communications in Computer and Information Science 2018; 919: 387-398. DOI: 10.1007/978-3-319-99447-5.

[6] Ayriyan AS, Ayrjan EA, Egorov AA, Maslyanitsyn IA, Shigorin VD. Numerical modeling of the static electric field effect on the director of the nematic liquid crystal director. Mathematical Models and Computer Simulations 2018; 10(6): 714-720. DOI: 10.1134/S2070048218060029.

[7] Ayriyan AA, Ayrjan EA, Egorov AA, Hadjichristov GB, Marinov YG, Maslyanitsyn IA, Petrov AG, Pribis J, Popova L, Shigorin VD, Strigazzi A, Torgova SI. Some features of second harmonic generation in the nematic liquid crystal $5 \mathrm{CB}$ in the pulsed-periodic electric field. 
Physics of Wave Phenomena 2016; 24(4): 259-267. DOI: 10.3103/S1541308X16040026.

[8] Ayriyan AA, Ayrjan EA, Egorov AA, Dencheva-Zarkova M, Hadjichristov GB, Marinov YG, Maslyanitsyn IA, Petrov AG, Popova L, Shigorin VD, Strigazzi A, Torgova SI. Modeling of static electric field effect on nematic liquid crystal director orientation in side-electrode cell. European Physical Journal WoC 2018; 173: 03002. DOI: 10.1051/epjconf/201817303002.

[9] Beeckman J, Yang T-H, Nys I, George JP, Lin T-H, Neyts K. Multi-electrode tunable liquid crystal lenses with one lithography step. Opt Lett 2018; 43(2): 271-274. DOI: 10.1364/OL.43.000271.

[10] Gilardi G, Asquini R, d'Alessandro A, Assanto G. Widely tunable electro-optic distributed Bragg reflector in liquid crystal waveguide. Opt Express 2010; 18(11): 1152411529. DOI: 10.1364/OE.18.011524.

[11] Wang T-J, Yang S-C, Chen T-J, Chen B-Y. Wide tuning of $\mathrm{SiN}$ microring resonators by auto-realigning nematic liquid crystal. Opt Express 2012; 20(14): 15853-15858. DOI: 10.1364/OE.20.015853.

[12] Liu J-M. Photonic devices. Cambridge: Cambridge University Press; 2005. ISBN: 978-0-521-55195-3.

[13] Rigneault H, Lourtioz J-M, Delalande C, Levenson A, eds. Nanophotonics. ISTE Ltd; 2006. ISBN: 978-1-905209-28-6.

[14] Hunsperger RG. Integrated optics. Theory and technology. New York: Springer-Verlag; 1984.

[15] Marcuse D. Light transmission optics. Van Nostrand Reinhold; 1972.

[16] Snyder AV, Love JD. Optical waveguide theory. Springer Science \& Business Media; 2012. ISBN: 978-0-41209950-2.

[17] Egorov AA. Correct investigation of the statistic irregularities of integrated optical waveguides with the use of the waveguide light scattering. Las Phys Letters 2004; 1(8): 421-428. DOI: 10.1002/lapl.200310094.

[18] Egorov AA. Theory of laser radiation scattering in integrated optical waveguide with 3D-irregularities in presence of noise: vector consideration. Las Phys Letters 2004; 1(12): 579-585. DOI: 10.1002/lapl.200410140.

[19] Egorov AA, Sevastyanov LA. Mode structure of smoothly irregular integrated optical four-layer three-dimensional waveguide. Quant Electron 2009; 39(6): 566-574. DOI: 10.1070/QE2009v039n06ABEH013966.

[20] Egorov AA, Lovetskiy KP, Sevastyanov AL, Sevastianov LA. Modelling of guided modes (eigenmodes) and synthesis of thin film generalized waveguide Luneburg lens in the zero-order vector approximation. Quant $\begin{array}{llll}\text { Electron } & 2010 ; & 40(9): & 830-836 .\end{array}$ 10.1070/QE2010v040n09ABEH014332.

[21] Egorov AA. Theoretical, experimental and numerical methods for investigating the characteristics of laser radiation scattered in the integrated-optical waveguide with three-dimensional irregularities. Quantum Electronics 2011; 41(7): 644-649. DOI: 10.1070/QE2011v041n07ABEH014560.

[22] Egorov AA. Study of bifurcation processes in a multimode waveguide with statistical irregularities. Quantum Electronics 2011; 41(10): 911-916. DOI: 10.1070/QE2011v041n10ABEH014683.
[23] Egorov AA. Theoretical and numerical analysis of propagation and scattering of eigen- and non-eigenmodes of an irregular integrated-optical waveguide. Quantum Electronics 2012; 42(4): 337-344. DOI: 10.1070/QE2012v042n04ABEH014809.

[24] Egorov AA. Numerical investigation of characteristics of laser radiation scattered in an integrated optical waveguide with three-dimensional inhomogeneities. Optics and Spectroscopy 2012; 112(2): 280-290. DOI: 10.1134/S0030400X12020105.

[25] Sevastyanov LA, Egorov AA, Sevastyanov AL. Method of adiabatic modes in studying problems of smoothly irregular open waveguide structures. Physics of Atomic Nuclei 2013; 76(2): 224-239. DOI: 10.1134/S1063778813010134.

[26] Egorov AA, Sevast'yanov LA, Sevast'yanov AL. Method of adiabatic modes in research of smoothly irregular integrated optical waveguides: zero approximation. Quantum Electronics 2014; 44(2): 167173. DOI: 10.1070/QE2014v044n02ABEH015303.

[27] Egorov AA, Lovetskiy KP, Sevastyanov AL, Sevastyanov LA. Integrated Optics: Theory and Computer Modelling. A monograph. Izdatel'stvo RUDN; 2015 (in Russian). ISBN: 978-5-209-06615-6.

[28] Born M, Wolf E. Principles of optics. Pergamon Press; 1986. ISBN: 0-08-026482-4.

[29] Goodman JW. Introduction to Fourier optics. McGrawHill; 1996. ISBN: 0-07-024254-2.

[30] Yong M. Optics and lasers: Including fibers and optical waveguides. Springer; 2001. ISBN: 978-3-540-65741-5.

[31] Ayriyan AA, Ayryan EA, Dencheva-Zarkova M, Egorov AA, Hadjichristov GB, Marinov YG, Maslyanitsyn IA, Petrov AG, Popova L, Shigorin VD, Torgova SI. Simulation of the static electric field effect on the director orientation of nematic liquid crystal in the transition state. Physics of Wave Phenomena 2019; 27(1): 67-72. DOI: 10.3103/S1541308X19010114.

[32] Egorov AA, Egorov MA, Stavtsev AV, Timakin AG, Chekhlova TK. A fast integrated optical sensor of gaseous substances. Journal of Russian Laser Research 2010; 31(1): 12-21. DOI: 10.1007/s10946-010-9120-z.

[33] Zografopoulos DC, Beccherelli R. Long-range plasmonic directional coupler switches controlled by nematic liquid crystals. Opt Express 2013; 21(7): 8240-8250. DOI: 10.1364/OE.21.008240.

[34] Wang Y, Li H, Zhao L, Liu Y, Liu S, Yang J. Tapered optical fiber waveguide coupling to whispering gallery modes of liquid crystal microdroplet for thermal sensing application. Opt Express 2017; 25(2): 918-926. DOI: 10.1364/OE.25.000918.

[35] Morgan SP. General solution of the Luneberg lens problem. J Appl Phys 1958; 29(9): 1358-1368. DOI: 10.1063/1.1723441.

[36] Southwell WH. Index profiles for generalized Luneburg lenses and their use in planar optical waveguides. J Opt Soc A 1977; 67(8): 1010-1014.

[37] Colombini E. Design of thin-film Luneburg lenses for maximum focal length control. Appl Opt 1981; 20(20): 3589-3593. DOI: 10.1364/AO.20.003589.

[38] Falco ADi, Kehr SC, Leonhardt U. Luneburg lens in silicon photonics. Opt Express 2011; 19(6): 5156-5162. DOI: 10.1364/OE.19.005156.

\section{Authors' information}

Aleksandr Alekseyevich Egorov (b. 1956), Dr.Sc., graduated from Peoples’ Friendship University of Russia in 1984, majoring in Radiophysics. He is a member of the Presidium of Moscow's A.S. Popov Scientific-Technical, Radiotechniques, Electronics and Communication Society. He is a member of the International Telecommunication Academy. Currently he works as a Leading Research Fellow at A.M. Prokhorov General Physics Institute, Russian Academy 
of Sciences. Research interests include: integrated optics, quantum electronics, radiophysics, integrated-optical sensors, nonlinear optics, nanophotonics, computer modeling. E-mail: yegorov@kapella.gpi.ru.

Leonid Antonovich Sevastianov (b. 1949), Dr.Sc., graduated from Peoples' Friendship University of Russia in 1971, majoring in Physics. Currently he works as a Professor at Applied Probability and Informatics department of Peoples' Friendship University of Russia, and as a Leading Researcher in the Bogoliubov Laboratory of Theoretical Physics at the Joint Institute for Nuclear Research. Research interests include: mathematical modeling, integrated optics, quantum mechanics, numerical methods. E-mail: sevastianov-la@,rudn.ru.

Vladimir Dmitrievich Shigorin (b. 1944), PhD, graduated from Lomonosov Moscow State University in 1967, majoring in Physics. Currently he works as a Leading Research Fellow at A.M. Prokhorov General Physics Institute, Russian Academy of Sciences (GPI of RAS). Research interests include: nonlinear optics, thin films physics, nanophotonics. E-mail: shigorin@ran.gpi.ru.

Alexander Serzhikovich Ayriyan (b. 1983), graduated from the MIREA - Russian Technological University in 2007, majoring in Computers, Systems and Networks. Currently he works as a Research Fellow in the Laboratory of Information Technology of the Joint Institute for Nuclear Research in Dubna, and in A.I. Alikhanyan National Science Laboratory in Yerevan, Armenia. Research interests include: mathematical modeling of physical systems, numerical methods, difference schemes, parallel programming. E-mail: ayriyan@jinr.ru.

Edik Artashevich Ayriyan (b. 1951), PhD, graduated from Yerevan State University in 1973, majoring in Mathematics. Currently he works as a Head of Sector in the Laboratory of Information Technology of the Joint Institute for Nuclear Research in Dubna. Research interests include: mathematical modeling, numerical methods, nonlinear problems, multigrid methods, parallel algorithms. E-mail: ayrjan@jinr.ru.

Received April 24, 2019. The final version - June 22, 2019. 